Christina W. Hilmarsen ${ }^{1,9}$, Sarah Wilke ${ }^{2,9}$, Harald Engan ${ }^{1,3}$, Martijn A. Spruit ${ }^{2}$, Johan Rodenburg ${ }^{1}$, Daisy J.A. Janssen ${ }^{2}$, Sigurd Steinshamn ${ }^{1,4,5}$, Paul W. Jones ${ }^{6}$, Emiel F.M. Wouters ${ }^{2,7}$, Line Oldervoll ${ }^{1,8,10}$ and Frits M.E. Franssen ${ }^{2,10}$

${ }^{1}$ LHL Health Røros, Norwegian Heart and Lung Patient Organization, Røros, ${ }^{4}$ Lung Dept, St Olav's University Hospital, Trondheim, ${ }^{5}$ K.G. Jebsen Center of Exercise in Medicine, Dept of Circulation and Medical Imaging, Norwegian University of Technology and Science, Trondheim, and ${ }^{8}$ Centre for Health Promotion, Institute for Social Work and Health Science, Norwegian University of Technology and Science, Trondheim, Norway. ${ }^{2}$ Program Development Centre, CIRO+, Centre of Expertise for Chronic Organ Failure, Horn, and ${ }^{7}$ Dept of Respiratory Medicine, Maastricht University Medical Centre+ (MUMC+), Maastricht, The Netherlands. ${ }^{3}$ Dept of Engineering and Sustainable Development, Mid Sweden University, Östersund, Sweden. ${ }^{6}$ Division of Clinical Science, St George's University of London, London, UK. ${ }^{9}$ Both authors contributed equally. ${ }^{10}$ Both authors contributed equally.

Correspondence: C.W. Hilmarsen, Research and Development Group, LHL Helse Røros, Øverhagaen 15, N-7374 Røros, Norway. E-mail: cwhi@lhl-helse.no

Received: Sept 182013 | Accepted: Sept 182013 | First published online: Oct 102013

Support statement: The collection of the Dutch data was financially supported by the Lung Foundation Netherlands (3.4.10.015) and GlaxoSmithKline (SCO115406).

Conflict of interest: Disclosures can be found alongside the online version of this article at www.erj.ersjournals.com

\title{
References
}

Janssen DJ, Spruit MA, Leue C, et al. Symptoms of anxiety and depression in COPD patients entering pulmonary rehabilitation. Chron Respir Dis 2010; 7: 147-157.

2 Lou P, Zhu Y, Chen P, et al. Prevalence and correlations with depression, anxiety, and other features in outpatients with chronic obstructive pulmonary disease in China: a cross-sectional case control study. BMC Pulm Med 2012; 12: 53 .

3 Jones PW, Quirk FH, Baveystock CM, et al. A self-complete measure of health status for chronic airflow limitation. The St. George's Respiratory Questionnaire. Am Rev Respir Dis 1992; 145: 1321-1327.

4 Jones PW, Harding G, Berry P, et al. Development and first validation of the COPD Assessment Test. Eur Respir J 2009; 34: 648-654.

5 Vestbo J, Hurd SS, Agusti AG, et al. Global strategy for the diagnosis, management and prevention of chronic obstructive pulmonary disease: GOLD executive summary. Am J Respir Crit Care Med 2013; 187: 347-365.

6 Sillen MJ, Franssen FM, Delbressine JM, et al. Heterogeneity in clinical characteristics and co-morbidities in dyspneic individuals with COPD GOLD D: findings of the DICES trial. Respir Med 2013; 107: 1186-1194.

Zigmond AS, Snaith RP. The hospital anxiety and depression scale. Acta Psychiatr Scand 1983; 67: 361-370.

Papaioannou AI, Bartziokas K, Tsikrika S, et al. The impact of depressive symptoms on recovery and outcome of hospitalised COPD exacerbations. Eur Respir J 2013; 41: 815-823.

9 Kunik ME, Roundy K, Veazey C, et al. Surprisingly high prevalence of anxiety and depression in chronic breathing disorders. Chest 2005; 127: 1205-1211.

10 Lee YS, Park S, Oh YM, et al. Chronic obstructive pulmonary disease assessment test can predict depression: a prospective multi-center study. J Korean Med Sci 2013; 28: 1048-1054.

\section{Molecular epidemiological analysis suggests cross-infection with Pseudomonas aeruginosa is rare in non-cystic fibrosis bronchiectasis}

\section{To the Editor:}

In both cystic fibrosis (CF) and non-CF bronchiectasis (NCFBr) chronic Pseudomonas aeruginosa infection is adversely prognostic $[1,2]$. In $\mathrm{CF}$, epidemic infections with specific clones of $P$. aeruginosa are associated with further adverse outcomes [3,4]. This cross-infection risk has led to segregation of patients [5]. There are few data on $P$. aeruginosa cross-infection in NCFBr. As a result, segregation in NCFBr has not been addressed in guidelines [6]. 
Our aim was to undertake a cross-infection study in NCFBr. This was undertaken in an adult bronchiectasis service in the north-east of England (UK) that is separated from the regional CF unit (sited 2 miles $(3 \mathrm{~km})$ away). The service was initiated in 2007 with a weekly specialist clinic without a Pseudomonas-specific clinic. When NCFBr patients are hospitalised, there is a preference for cubicle-based (single-patient room) management, but when cubicles are unavailable, patients are managed in six-bed bays. All patients had computed tomographic confirmation and had predominantly idiopathic or post-infectious bronchiectasis with CF excluded following current guidelines [6]. The study had ethical permission and Caldicott approval (Newcastle and North Tyneside National Research Ethics Service Committee).

56 isolates were selected for analysis. Six were chosen from CF patients as laboratory controls. 50 were NCFBr isolates collected between 2008 and 2011 from 40 NCFBr patients. 36 patients (patients 1-36) attending the adult bronchiectasis service were randomly selected. Longitudinal isolates were included from 10 patients with a mean (range) 16 months (2-35 months) between isolates. Additionally, single isolates were chosen from four NCFBr patients (patients 37-40) as potential patient controls who had not attended the specialist clinic and had not been hospitalised. Isolates were cultured from spontaneous sputum and identified as $P$. aeruginosa by routine biochemical methods and matrix-assisted laser desorption/ionisationtime-of-flight (MALDI-TOF) mass spectrometry.

We used two genotyping methods; in each case, testing was blinded. First, we used the ArrayTube (AT genotyping; Alere, Jena, Germany) as per the manufacturer's protocol. This system detects 13 singlenucleotide polymorphisms within conserved $P$. aeruginosa genes and the presence/absence of 38 variable genetic markers in the accessory genome. The data were converted into a four-digit hexadecimal code and then compared to a database [7,8]. Secondly, we used variable number tandem repeat (VNTR) analysis based on variation in the number of DNA repeats at specified sites across the genome at nine variable loci [9] with comparison to a VNTR database [10].

The vast majority of the $36 \mathrm{NCFBr}$ patients attending the service (patients 1-36) harboured their own strains (fig. 1). Patients 11 and 27 had distinct variants of clone C, which is a lineage that is widely found globally [10]; two patients in the nonhospitalised control group also had further variants of this clone (patients 39 and 40). Among the 34 remaining NCFBr patients attending the service, there were three pairs (patients 2 and 34; 12 and 19; and 16 and 17) whose isolates of P. aeruginosa shared very similar profiles by at least one method (fig. 1). For patients 12 and 19, this was confirmed by pulsed-field gel electrophoresis of SpeI-digested genomic DNA; this is an unusual profile and that these patients shared the same strain almost certainly does reflect cross-infection. Patients 2 and 34 both had a widely found type belonging to sequence type 27, while patients 16 and 17 had clearly distinct strains by VNTR, suggesting only one probable case of cross-infection.

The location of the NCFBr isolates among the wider population structure of P. aeruginosa, based on AT genotype, was mapped (not shown) and demonstrated that $\mathrm{NCFBr}$ isolates were widely distributed. AT analysis found several pattern matches with the AT database: matches were with AT clones A2, K, E, I, A7, A, L, J, A5 and U. Both methods correctly identified the known control strains from CF patients.

Longitudinally paired isolates from 10 patients were examined (patients 3, 6-11, 14, 16 and 18). For nine patients, the first and second isolates were indistinguishable by both AT and VNTR genotyping, confirming persistence of the initial strain. In one patient (patient 11), VNTR analysis indicated that both isolates were identical, but AT genotyping did not.

Our key finding was the absence of dominant clones of $P$. aeruginosa with little evidence of cross-infection and many strains that were "unique". While there is distinct clustering of AT clone types in ocular keratitis, the isolates from NCFBr were widely distributed in the Pseudomonas population structure. Collectively, this suggests that sporadic infection is the most common mode of infection in NCFBr. Clone $\mathrm{C}$ was found in only $6 \%$ of the NCFBr patients and is known to be widely distributed within the environment [8]. Crossinfection with clone C seems unlikely, as the VNTR profiles varied at two or three loci, suggesting that they were independently acquired. Other isolates matched those previously isolated from various clinical and environmental sources [8]. These data may suggest a risk of environmental acquisition in $\operatorname{NCFBr}[5,8]$.

The absence of a dominant $P$. aeruginosa clone in NCFBr may reflect many factors, including the lower incidence of $P$. aeruginosa infection in $\mathrm{NCFBr}(<50 \%)$ as compared to $\mathrm{CF}(\leqslant 80 \%)$. The probable lower rate of hospitalisation in NCFBr compared to CF may also limit cross-infection exposures. The standard infection prevention and control measures used in our NCFBr centre are less stringent than those implemented in designated CF centres. The absence of significant cross-infection herein is therefore unlikely to be due to a higher standard of infection control practices.

Notably, our study is solely focused on $P$. aeruginosa cross-infection and we cannot exclude cross-infection in the NCFBr population with Staphylococcus aureus, Haemophilus influenzae or nontuberculous 
Patient Hexadecimal code

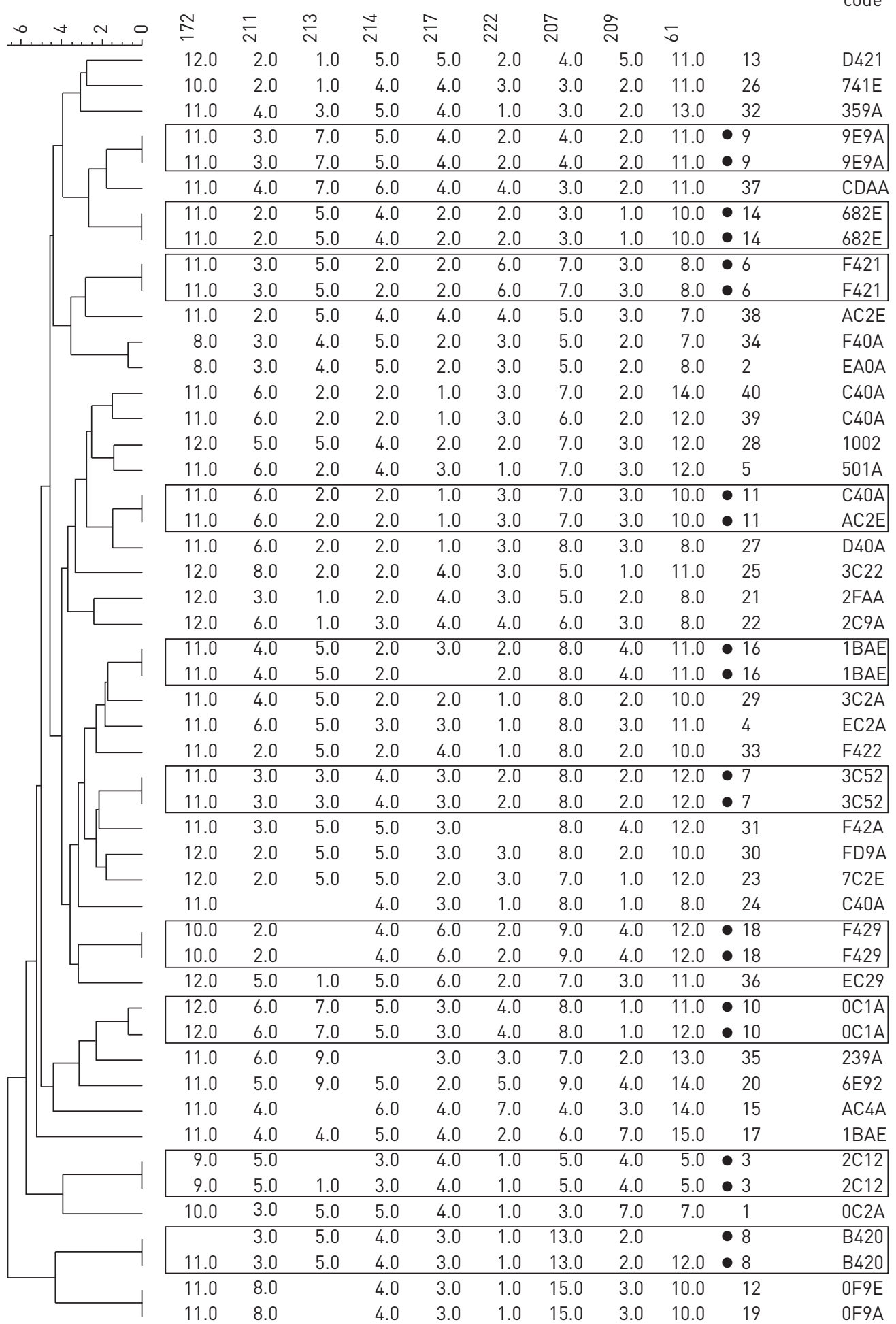

FIGURE 1 Variable number tandem repeat dendrogram showing the diversity of types among the 50 non-cystic fibrosis bronchiectasis isolates analysed. The similarity coefficient was calculated using Euclidian distance, and strains were clustered using the unweighted pair group method with arithmatic mean using BioNumerics software (Applied Maths, Sint-Martens-Latem, Belgium). Pairs of longitudinal isolates are marked in boxes and with a dot. The hexadecimal code derived by ArrayTube analysis (Alere, Jena, Germany) is included for reference, as are the patient numbers (see text). 
mycobacteria. Furthermore, limitations of our study include the single-centre design. Our observations may have been different if our NCFBr centre facilities were shared with our regional CF clinic. We therefore advocate further multicentre studies to delineate the cross-infection risks.

0 @ERSpublications

Cross-infection with Pseudomonas aeruginosa is rare in non-cystic fibrosis bronchiectasis http://ow.ly/sjvKz

Anthony De Soyza ${ }^{1,2}$, Audrey Perry ${ }^{3}$, Amanda J. Hall ${ }^{4}$, Syba S. Sunny ${ }^{2}$, Kathy E. Walton ${ }^{3}$, Nazim Mustafa ${ }^{5}$, Jane Turton ${ }^{5}$, Dervla T. Kenna ${ }^{5}$ and Craig Winstanley ${ }^{4}$

${ }^{1}$ Institute of Cellular Medicine, Newcastle University, Newcastle upon Tyne, ${ }^{2}$ Sir William Leech Centre for Respiratory Research and Freeman Hospital Adult Bronchiectasis Unit, Newcastle upon Tyne Hospitals NHS Foundation Trust, Newcastle upon Tyne, ${ }^{3}$ Dept of Medical Microbiology, Newcastle upon Tyne Hospitals NHS Foundation Trust, Newcastle upon Tyne, ${ }^{4}$ Institute of Infection and Global Health, University of Liverpool, Liverpool, and ${ }^{5}$ Antimicrobial Resistance and Healthcare Associated Infections Reference Unit, Public Health England, London, UK.

Correspondence: A. De Soyza, Institute of Cellular Medicine, Sir William Leech Building, The Medical School, Newcastle University, Newcastle upon Tyne, NE2 4HH, UK. E-mail: anthony.de-soyza@ncl.ac.uk

Received: Sept 252013 | Accepted: Oct 162013 | First published online: Oct 312013

Support statement: A. De Soyza acknowledges a Higher Education Funding Council for England senior lectureship and funding from the National Institute for Health Research Newcastle Biomedical Resource Centre for Ageing and Chronic Disease.

Conflict of interest: Disclosures can be found alongside the online version of this article at www.erj.ersjournals.com

\section{References}

1 Chalmers JD, Goeminne P, Aliberti S, et al. The Bronchiectasis Severity Index: an international derivation and validation study. Am J Respir Crit Care Med 2013 [In press DOI: 10.1164/rccm.201309-1575OC].

2 Emerson J, Rosenfeld M, McNamara S, et al. Pseudomonas aeruginosa and other predictors of mortality and morbidity in young children with cystic fibrosis. Pediatr Pulmonol 2002; 34: 91-100.

3 Al-Aloul M, Crawley J, Winstanley C, et al. Increased morbidity associated with chronic infection by an epidemic Pseudomonas aeruginosa strain in CF patients. Thorax 2004; 59: 334-336.

4 Cheng K, Smyth RL, Govan JR, et al. Spread of $\beta$-lactam-resistant Pseudomonas aeruginosa in a cystic fibrosis clinic. Lancet 1996; 348: 639-642.

$5 \quad$ Fothergill JL, Walshaw MJ, Winstanley C. Transmissible strains of Pseudomonas aeruginosa in cystic fibrosis lung infections. Eur Respir J 2012; 40: 227-238.

6 Pasteur MC, Bilton D, Hill AT, et al. British Thoracic Society guideline for non-CF bronchiectasis. Thorax 2010; 65: Suppl. 1, i1-i58.

7 Stewart RM, Wiehlmann L, Ashelford KE, et al. Genetic characterization indicates that a specific subpopulation of Pseudomonas aeruginosa is associated with keratitis infections. J Clin Microbiol 2011; 49: 993-1003.

8 Wiehlmann L, Wagner G, Cramer N, et al. Population structure of Pseudomonas aeruginosa. Proc Natl Acad Sci USA 2007; 104: 8101-8106.

9 Turton JF, Turton SE, Yearwood L, et al. Evaluation of a nine-locus variable-number tandem-repeat scheme for typing of Pseudomonas aeruginosa. Clin Microbiol Infect 2010; 16: 1111-1116.

10 Martin K, Baddal B, Mustafa N, et al. Clusters of genetically similar isolates of Pseudomonas aeruginosa from multiple hospitals in the UK. J Med Microbiol 2013; 62: 988-1000.

\section{Rapidly progressing tuberculosis outbreak in a very low risk group}

To the Editor:

We report an unusual tuberculosis (TB) outbreak, centred on a professional basketball team in Montevideo, Uruguay, a country with a low TB incidence (21 cases per 100000$)$. Uruguay has a relatively high income (2010 gross domestic product USD14338 per capita), a low HIV prevalence (305 cases per 100000 ) and a very low rate of multidrug-resistant $\mathrm{TB}(0.24 \%)$ [1]. In August 2008, a young male member of the basketball team was diagnosed with TB, with positive microscopic examination and Ziehl-Nielsen staining of a sputum sample (graded 1). Chest radiography indicated a bilateral pulmonary form with cavities. TB was bacteriologically confirmed 20 days later, with a Mycobacterium tuberculosis isolate pan-susceptible to antituberculous drugs. The patient was compliant with first-line treatment $\left(2 \mathrm{HRZ} / 4 \mathrm{H}_{2} \mathrm{R}_{2}: 2\right.$ months daily 